\title{
An Earth-mass Planet in a 1-AU Orbit around an Ultracool Dwarf
}

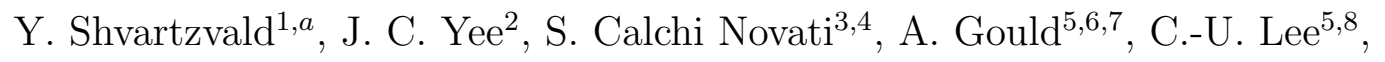 \\ and \\ C. Beichman ${ }^{9}$, G. Bryden ${ }^{1}$, S. Carey ${ }^{10}$, B. S. Gaudi ${ }^{7}$, C. B. Henderson ${ }^{1, a}$, W. Zhu ${ }^{7}$ \\ (Spitzer team) \\ and

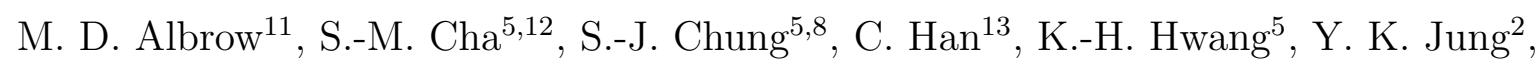 \\ D.-J. Kim ${ }^{5}$, H.-W. Kim ${ }^{5}$, S.-L. Kim ${ }^{5,8}$, Y. Lee ${ }^{5,12}$, B.-G. Park ${ }^{5,8}$, R. W. Pogge ${ }^{7}$, Y.-H. Ryu ${ }^{5}$, \\ I.-G. Shin ${ }^{2}$ \\ (KMTNet group)
}

\begin{abstract}
We combine Spitzer and ground-based KMTNet microlensing observations to identify and precisely measure an Earth-mass $\left(1.43_{-0.32}^{+0.45} M_{\oplus}\right)$ planet OGLE-2016BLG-1195Lb at $1.16_{-0.13}^{+0.16} \mathrm{AU}$ orbiting a $0.078_{-0.012}^{+0.016} M_{\odot}$ ultracool dwarf. This is the
\end{abstract}

\footnotetext{
${ }^{1}$ Jet Propulsion Laboratory, California Institute of Technology, 4800 Oak Grove Drive, Pasadena, CA 91109, USA

${ }^{2}$ Smithsonian Astrophysical Observatory, 60 Garden St., Cambridge, MA 02138, USA

${ }^{3}$ IPAC, Mail Code 100-22, Caltech, 1200 E. California Blvd., Pasadena, CA 91125, USA

${ }^{4}$ Dipartimento di Fisica "E. R. Caianiello", Università di Salerno, Via Giovanni Paolo II, 84084 Fisciano (SA), Italy

${ }^{5}$ Korea Astronomy and Space Science Institute, Daejon 305-348, Republic of Korea

${ }^{6}$ Max-Planck-Institute for Astronomy, Königstuhl 17, 69117 Heidelberg, Germany

${ }^{7}$ Department of Astronomy, Ohio State University, 140 W. 18th Ave., Columbus, OH 43210, USA

${ }^{8}$ Korea University of Science and Technology, 217 Gajeong-ro, Yuseong-gu, Daejeon 34113, Korea

${ }^{9}$ NASA Exoplanet Science Institute, California Institute of Technology, Pasadena, CA 91125, USA

${ }^{10}$ Spitzer, Science Center, MS 220-6, California Institute of Technology,Pasadena, CA, USA

${ }^{11}$ University of Canterbury, Department of Physics and Astronomy, Private Bag 4800, Christchurch 8020, New Zealand

${ }^{12}$ School of Space Research, Kyung Hee University, Yongin 446-701, Republic of Korea

${ }^{13}$ Department of Physics, Chungbuk National University, Cheongju 361-763, Republic of Korea

aNASA Postdoctoral Program Fellow
} 
lowest-mass microlensing planet to date. At $3.91_{-0.46}^{+0.42} \mathrm{kpc}$, it is the third consecutive case among the Spitzer "Galactic distribution" planets toward the Galactic bulge that lies in the Galactic disk as opposed to the bulge itself, hinting at a skewed distribution of planets. Together with previous microlensing discoveries, the seven Earth-size planets orbiting the ultracool dwarf TRAPPIST-1, and the detection of disks around young brown dwarfs, OGLE-2016-BLG-1195Lb suggests that such planets might be common around ultracool dwarfs. It therefore sheds light on the formation of both ultracool dwarfs and planetary systems at the limit of low-mass protoplanetary disks.

Subject headings: gravitational lensing: micro - binaries: general - planetary systems - Galaxy: bulge

\section{Introduction}

Formation theories suggest that planets form in protoplanetary disks around their hosts, either through core accretion (e.g., Ida \& Lin 2005) or disk instability (e.g., Boss 2006). The masses and frequencies of planets are thus tightly related to the disk masses, and indirectly to the mass of their hosts. Brown dwarfs (BDs) are substellar objects not massive enough to ignite hydrogen fusion. According to current theory, BDs represent the lower-mass end of star formation via direct collapse of molecular clouds (e.g., Luhman 2012). Therefore, studying planet formation around BDs, with their low-mass disks, probes the limiting conditions for planet formation. Payne \& Lodato (2007) extended the study of core accretion models of Ida \& Lin (2005) to ultracool dwarfs (very low-mass stars and BDs). They predict that if BDs have few-Jupiter-mass disks, then Earth-mass planets should be frequent around them, with typical semi-major axes $\sim 1 \mathrm{AU}$ and maximum planetary masses $\sim 5 M_{\oplus}$. However, if BD disks contain only a fraction of a Jupiter mass then they probably cannot form even an Earth-mass planet.

Early statistical studies of disks around ultracool dwarfs showed that they are as frequent as around Sun-like stars (Apai 2013). More recently, Testi et al. (2016) used ALMA to study 17 young brown dwarfs and found continuum emission, indicating the existence of dusty disks, in 11 of them. The estimated dust masses in these disks are $\sim 0.5-6 M_{\oplus}$, suggesting total disk masses $\sim 0.1-2 M_{J}$. Daemgen et al. (2016) used Herschel to study 29 ultracool dwarfs and found that about half have disks with at least one Jupiter mass.

The detection of planets around ultracool dwarfs is challenging for most planetary detection methods because they rely on light from the faint host and/or the even fainter planet. 
Four such systems were found via direct imaging, all with a massive planet $\left(>4 M_{J}\right)$ : 2 MASS 1207-3932 (Chauvin et al. 2004), 2MASS 0441-2301 (Todorov et al. 2010), VHS 1256-1257 Gauza et al. 2015), and CFBDSIR 1458+1013 (Liu et al. 2011). However, the companionstar mass ratios $(q>0.15)$ and large separations ( $>15 \mathrm{AU}$, except CFBDSIR $1458+1013$ with $\sim 2.3$ AU but $q \sim 0.5)$ suggest they were formed similarly to binary systems through gravitational fragmentation (Lodato et al. 2005) rather than like planetary systems. Recently, Gillon et al. (2017) detected seven terrestrial planets transiting the nearby ultracool dwarf star TRAPPIST-1, whose host mass $0.080 \pm 0.007 M_{\odot}$ places it slightly above the hydrogen burning limit. The planets have few-day near-resonant periods, suggesting they formed farther out and then migrated inward.

In contrast to these discovery techniques, gravitational microlensing does not rely on light from the system but is directly sensitive to the masses of the planet and its host. Its basic observable is the Einstein timescale $t_{\mathrm{E}}$, which encompasses the total lens mass $M$, the lens-source relative parallax $\pi_{\text {rel }}$, and relative proper motion $\boldsymbol{\mu}_{\text {geo }}$. Breaking the $\left(M, \pi_{\text {rel }}, \mu_{\text {geo }}\right)$ degeneracy requires two additional parameter measurements, the angular Einstein radius $\theta_{\mathrm{E}}$ and the amplitude of the microlens parallax vector $\pi_{\mathrm{E}}=\left|\boldsymbol{\pi}_{\mathrm{E}}\right|$, which yield (Gould 1992 , 2000):

$$
M=\frac{\theta_{\mathrm{E}}}{\kappa \pi_{\mathrm{E}}} ; \quad \pi_{\mathrm{rel}}=\pi_{\mathrm{E}} \theta_{\mathrm{E}} ; \quad \boldsymbol{\mu}_{\mathrm{geo}}=\frac{\theta_{\mathrm{E}}}{t_{\mathrm{E}}} \frac{\boldsymbol{\pi}_{\mathrm{E}, \mathrm{geo}}}{\pi_{\mathrm{E}}},
$$

where $\kappa \equiv \frac{4 G}{c^{2} \mathrm{AU}} \simeq 8.14 \frac{\mathrm{mas}}{M_{\odot}}$.

Four previous microlensing events revealed planets orbiting ultracool dwarfs. Bennett et al. (2008) found a low-mass planet in event MOA-2007-BLG-192 whose characterization was then substantially tightened by Gould et al. (2010) and Kubas et al. (2012). The latter used adaptive optics to measure the lens flux, from which they derived a planet of mass $\sim 3 M_{\oplus}$ projected at $\sim 0.7 \mathrm{AU}$ around a $\sim 0.08 M_{\odot}$ late $\mathrm{M}$ dwarf. However, because the planetary deviation was sparsely covered, the uncertainties on the planet's mass and separation are large. Gould et al. (2014) discovered a $\sim 2 M_{\oplus}$ planet at $\sim 0.8$ AU from a 0.1$0.15 M_{\odot} \mathrm{M}$ dwarf, which is one member of a binary system with a slightly heavier companion $\left(0.12-0.18 M_{\odot}\right)$. This planet had the lowest securely-measured mass found by microlensing previous to the one presented here. These two detections of planets with $\sim 1 M_{\oplus}$ at $\sim 1 \mathrm{AU}$ around ultracool dwarfs agree well with the predictions of Payne \& Lodato (2007).

In addition, Han et al. (2013) discovered a $\sim 2 M_{J}$ planet at projected separation $\sim 0.9 \mathrm{AU}$ orbiting a $\sim 0.02 M_{\odot}$ BD. While the mass ratio suggests a binary-like formation mechanism, the relatively close projected separation hints at possible in-disk formation. Finally, Sumi et al. (2016) discovered a planetary system in microlensing event MOA-2013-BLG-605. They found three degenerate microlensing models, two of which suggest a super Earth orbiting at $1-2 \mathrm{AU}$ around a BD, while the third suggests a Neptune orbiting a $0.2 M_{\odot} \mathrm{M}$ dwarf. The 
mass ratio in all solutions clearly favor a planetary formation scenario, but the possibility of a mid-M-dwarf host prevents the setting of strong constraints on planet formation models around BDs.

Here we analyze the planetary microlensing event OGLE-2016-BLG-1195. The event was observed by Spitzer and several ground surveys. The ground light curve shows a very short anomaly after the peak, indicating a low mass planetary companion, while the parallax measurement from Spitzer allows us to determine that it is a $\sim 1 M_{\oplus}$ planet around an ultracool dwarf at the hydrogen burning limit. The microlens modeling yields eight degenerate solutions, which are well understood theoretically (Refsdal 1966; Griest \& Safizadeh 1998). However, again for well-understood reasons, all solutions imply similar physical properties for the system.

The Spitzer microlensing campaign has enabled the systematic measurement of the microlens parallax for well over 200 events over its first three seasons. These will enable the first measurement of the Galactic distribution of planets (Calchi Novati et al. 2015a; Zhu et al. 2017), a demographic regime that is currently uniquely explored by microlensing (see also Penny et al. 2016). The 2014 and 2015 Spitzer campaigns have already led to the publication of two other planetary systems (Udalski et al. 2015b; Street et al.|2016), both of which are located in the Galactic disk, similar to OGLE-2016-BLG-1195Lb.

\section{Observational data and reduction}

OGLE-2016-BLG-1195 was alerted on UT 13:37 27 June 2016 by the Optical Gravitational Lensing Experiment (OGLE), using the OGLE Early Warning System (Udalski et al. 2015a). The event was also observed by the Microlensing Observations in Astrophysics (MOA) collaboration (Sumi et al. 2003), which recognized and alerted the planetary anomaly at UT 15:45 29 June, less than two hours after it began. The analysis and data in this paper are completely independent from that derived from the OGLE and MOA datasets Bond et al. 2017).

\subsection{KMTNet observations}

The event was observed by Korea Microlensing Telescope Network (KMTNet; Kim et al. 2016) that employs $4 \mathrm{deg}^{2}$ cameras at three sites: CTIO/Chile, SAAO/South Africa, and SSO/Australia. At $(\mathrm{RA}, \mathrm{Dec})=(17: 55: 23.5,-30: 12: 26.1)(\mathrm{J} 2000.0)$, the event was in an overlapping region between three high-cadence $(25 \mathrm{~min})$ fields, for a combined cadence $\Gamma=7 \mathrm{hr}^{-1}$, which allowed for dense coverage of the short anomaly. These observations were carried out as part of the routine survey and were not influenced by the MOA alert. 
Most observations were in $I$ band, with additional sparse $V$ band observations for source characterization. KMTNet photometry was extracted using an adapted version of the pySIS

DIA software (Albrow et al. 2009) for the event modeling, and DoPhot (Schechter et al. 1993) for the source color information.

\subsection{Spitzer observations}

The event was announced by OGLE on a Monday morning (PDT), less than two hours before the Spitzer team had to submit the targets to observe in the coming week. Based on a preliminary model suggesting a high-magnification event, it was selected as a "secret" target (see details below). On the following morning (PDT), the team confirmed the model using new OGLE data, and immediately announced the event as a "subjective" Spitzer target on June 28 UT 15:33 (HJD'=7568.15), about a day before the anomaly and 3.7 days before the Spitzer observations actually started.

Spitzer observations are of targeted, on-going events and thus require well defined selection criteria to avoid biases toward planetary events. These selection criteria are fully described in Yee et al. (2015). Here we briefly summarize the three selection modes. When an event passes certain brightness and planet sensitivity criteria (as inferred from its pointlens model) at the target submission deadline, it is automatically selected as a Spitzer target and considered as "objective". However, if an event model suggests it will have high planet sensitivity, the team can select it "subjectively", even before it meets the criteria (if it ever does). This allows for early Spitzer observations and increases the overall campaign sensitivity for planets while still permitting an unbiased sample. Lastly, the team can select a promising event with a weakly constrained point-lens model as "secret". If the event turns out to be promising (but without any indications of a planetary perturbation), the team can select (and announce) it as "subjective", and it is then included in the final sample. OGLE-2016-BLG-1195 was such event. (If a "secret" event turns out to be unpromising, it is discarded and is not included in the final statistical sample.)

OGLE-2016-BLG-1195 was observed by Spitzer during the final three weeks of the 2016 campaign (July 2-24), with a cadence of 1 observation per day. Each epoch is composed of six 30-second dithered exposures using the $3.6 \mu \mathrm{m}$ channel on IRAC, and the data were reduced using the new algorithm for Spitzer crowded-field photometry (Calchi Novati et al. 2015b). The event was faint in Spitzer images, with a peak of $L \sim 15.7$ and baseline of $L \sim 17.1$. The reductions therefore required special handling. The precise astrometric position of the source was determined from KMTNet difference images at high magnification. Using this position in the analysis, the Spitzer photometry was significantly improved relative to photometry 
based on the catalog position of the apparent "star" at the base of the microlensing event.

\section{Light Curve Analysis}

\subsection{Ground-only microlensing model}

Standard binary-lens microlensing models require seven geometric parameters to calculate the magnification, $A(t)$. These include the three point-lens parameters $\left(t_{0}, u_{0}, t_{\mathrm{E}}\right)$ (Paczynśki 1986), the angular source radius $\theta_{*}$ scaled to $\theta_{\mathrm{E}}\left(\rho=\theta_{*} / \theta_{\mathrm{E}}\right)$, and three parameters for the companion: the mass ratio $q$, the instantaneous projected separation (scaled to $\left.\theta_{\mathrm{E}}\right) s$, and an angle $\alpha$, between the source trajectory and the binary axis in the lens plane. In addition, each dataset $i$, has two parameters $\left(f_{s, i}, f_{b, i}\right)$ representing the source flux and any additional blend flux:

$$
f_{i}(t)=f_{s, i} A(t)+f_{b, i}
$$

The light curve (Figure 1) has one short $(<3 \mathrm{hr}$ ) anomaly $\Delta t \simeq 8.5 \mathrm{hr}$ after the peak of an otherwise standard point-lens moderate-magnification $(A \simeq 19)$ microlensing event. Combined with the effective timescale $t_{\text {eff }} \equiv u_{0} t_{\mathrm{E}} \sim 13 \mathrm{hr}$, this indicates a planetary massratio companion close to the Einstein ring, aligned $\tan ^{-1} 13 / 8.5 \sim 57^{\circ}$ relative to the source trajectory. The single bump in the light curve implies that the source passes the single prong of the central caustic, which faces ("points toward") the planet in both close and wide topologies. We first solve for $s<1$ because this topology is unencumbered by additional caustics on this side of the central caustic. The $(s>1)$ solution will immediately follow from the close-wide degeneracy (Griest \& Safizadeh 1998).

We seed a Markov-chain Monte-Carlo (MCMC) with close companions $(s<1)$ at an angle of $\alpha=57^{\circ}$ and with mass ratios ranging $q=10^{-3}-10^{-6}$. To calculate the model magnifications near and during the anomaly, we employ contour integration (Gould \& Gaucherel 1997) with 10 annuli to allow for limb darkening. We adopt a linear coefficient $u(I)=0.526$ Claret 2000), based on the source type derived in Section 4.1. Far from the anomaly we employ limb-darkened multipole approximations (Pejcha \& Heyrovský 2009; Gould 2008). We find $(s, q)=\left(0.98,5.5 \times 10^{-5}\right)$, which is in the resonant-caustic regime. While the source does not cross the caustic, it passes close enough and over a narrow "shoulder" on the magnification surface to permit a clear measurement of the scaled source size, $\sigma(\ln \rho)=13 \%$ (Figure 2).

Finally, we seed the $(s>1)$ MCMC search at $q=5.5 \times 10^{-5}$ and at the boundary between the wide and resonant topologies. The result is $(s, q)=\left(1.09,5.6 \times 10^{-5}\right)$ for which 
the source passes over a narrow "saddle" between two marginally separated caustics (Figure 2). The $(s<1)$ and $(s>1)$ solutions prove fully degenerate.

\subsection{Satellite microlens parallax}

Observations of a microlensing event by two widely separated observers (e.g., from Earth and space) results in different observed light curves (Refsdal 1966; Gould 1994). Since the physical separation between the two observers $\left(\mathbf{D}_{\perp}\right)$ is known, modeling the two light curves directly yields the microlens parallax,

$$
\boldsymbol{\pi}_{\mathrm{E}}=\frac{\mathrm{AU}}{D_{\perp}}(\Delta \tau, \Delta \beta) ; \quad \Delta \tau=\frac{t_{0, \mathrm{sat}}-t_{0, \oplus}}{t_{\mathrm{E}}} ; \quad \Delta \beta= \pm u_{0, \mathrm{sat}}- \pm u_{0, \oplus},
$$

where the subscripts indicate parameters as measured from the satellite and Earth. However, due to the symmetry of the problem it usually suffers from a four-fold "satellite" degeneracy in $\Delta \beta$ (Equation (3)).

When including Spitzer, we re-run the MCMC process with all four possibilities for both the wide and close configurations. In addition, we include a constraint on the Spitzer source flux, $f_{s, \text { Spitzer }}$, derived from color-color regression (see Section 4.1). We find that indeed the $2 \times 4=8$ possible solutions are fully degenerate. The magnitude $\left|\boldsymbol{\pi}_{\mathrm{E}}\right|$ is roughly the same for all solutions since $\left|u_{0, \oplus}\right| \ll 1$ (Gould \& Yee 2012). Table 1 summarizes the derived model parameters and their uncertainties for all eight solutions.

The most striking feature of these solutions is the relatively large microlens parallax $\pi_{\mathrm{E}} \sim$ 0.45, which (combined with the small value of $\theta_{\mathrm{E}}$ ) will yield small masses for both the host and the planet (see Section 4.2). However, Figure1 demonstrates, independent of any model, that there is a significant parallax effect. First, inset (c) shows that the Spitzer magnification is larger than the ground-based magnification at the same time. Because the peak groundbased magnification is quite high $A_{\max , \oplus} \simeq u_{0, \oplus}^{-1} \simeq 19$, Spitzer's substantially higher postpeak magnification directly implies that it peaks substantially later. The fact that the first Spitzer points nearly overlap the ground-based magnification then implies substantially larger $u_{0, \text { Spitzer }}$ as well. One may therefore qualitatively infer that both components of the parallax should be large.

To make this argument more concrete, we consider a range of models with different $t_{0, \text { Spitzer }}=7569,7570, \ldots 7575$, and with the Spitzer flux constraint set at $-2 \sigma, 0 \sigma$, and $+2 \sigma$ from value that we derive from the $V I L$ color-color relation. The lowest of these value $t_{0, \text { Spitzer }}=7569$ would yield the lowest $\left|\Delta t_{0}\right|$ and therefore the lowest value of $\left|\pi_{\mathrm{E}, E}\right|$ (and so potentially the lowest value of $\pi_{\mathrm{E}}$, depending on the value of $\Delta \beta$ for that solution). This (and other low $t_{0, \text { Spitzer }}$ ) solutions also seem naively in accord with the fact that the data appear to fall almost monotonically over the first nine points, whereas the best model peaks 
between the second and third data points. The last value in this series is a few days later than in the best fit model.

The results of this test are shown in Figure 3. The lower panel demonstrates that indeed if $t_{0, \text { Spitzer }}$ is artificially pushed lower, the value of $\pi_{\mathrm{E}}$ is reduced. However, for $t_{0, \text { Spitzer }} \geq 7571$, this is almost entirely due to shrinking $\left|\pi_{\mathrm{E}, E}\right|$, while $\pi_{\mathrm{E}, N}$ remains essentially constant. Hence, at this point $\pi_{\mathrm{E}}$ is still $60 \%$ of the best-fit value (meaning that the main physical conclusions would be qualitatively similar), while $\Delta \chi^{2}=17.5$ is already quite high. Note also that the $\boldsymbol{\pi}_{\mathrm{E}}$ trajectories for different $f_{\text {s,Spitzer }}$ (shown by different colors) are essentially identical, with the major difference being the higher $\Delta \chi^{2}=4$ penalty for the $\pm 2 \sigma$ tracks.

The basic reason that $\pi_{\mathrm{E}}$ changes very little over this range is that, a week after the ground-based peak $(\tau, \beta)_{\oplus}=(0.7,0.05)$, while the "observed" Spitzer magnification is $A_{\text {Spitzer }}=3$, i.e., $u_{\text {Spitzer }}=0.35$. This implies $|\Delta \mathbf{u}|>0.35$ (hence $\pi_{\mathrm{E}}>\left(\mathrm{AU} / D_{\perp}\right)|\Delta \mathbf{u}|=$ 0.30) unless the model is forced to systematically fall below the Spitzer data.

\section{Physical properties}

The mass and distance of the lensing system can be derived from $\pi_{\mathrm{E}}$ and $\theta_{\mathrm{E}}$ (Equation 1). These allow us to use the mass ratio $q$ and the scaled projected separation $s$ to derive the masses and physical projected separation of the two bodies. While $\pi_{\mathrm{E}}$ is directly measured in the microlensing model, $\theta_{\mathrm{E}}$ is derived from $\rho$ (found from the light curve model) and $\theta_{*}$ (found using the color-magnitude diagram (CMD)): $\theta_{\mathrm{E}}=\theta_{*} / \rho$.

\section{1. $\mathrm{CMD}$}

The source properties are derived from its position on a CMD, constructed using stars from a $205^{\prime \prime} \times 205^{\prime \prime}$ field centered on the event's position (Figure 4), using KMTNet instrumental $V$-band and $I$-band magnitudes. We measure the centroid of the "red giant clump" (RGC) $(V-I, I)_{\mathrm{cl}, \mathrm{kmt}}=(-0.22,15.59)$ and compare it to the intrinsic centroid of $(V-I, I)_{\mathrm{cl}, 0}=(1.06,14.44)$ (Bensby et al. 2013; Nataf et al. 2013) for the event's Galactic coordinates $(l, b)=(0.0,-2.5)$. The KMTNet $I$-band source magnitude as inferred from the microlensing model is $I_{s, \mathrm{kmt}}=18.99 \pm 0.02$, and assuming it is behind the same dust column as the red clump, its intrinsic magnitude is $I_{s, 0}=17.84 \pm 0.04$.

A standard way to determine the instrumental $(V-I)_{s}$ color is from regression of $V$ versus $I$ flux as the source magnification changes (Gould et al. 2010). Applying this to KMTNet data yields $(V-I)_{s, \mathrm{kmt}}=-0.59 \pm 0.02$, from which we derive $(V-I)_{s, 0}=0.68 \pm 0.03$ by correcting to the clump offset found above. Using standard color-color relations (Bessell $\&$ Brett 1988) and the relation between angular source size and surface brightness Kervella 
et al. 2004), we find $\theta_{*}=0.82 \pm 0.07 \mu$ as.

We use red giant branch stars $\left(14.6<I_{\mathrm{KMT}}<16.6 ;-0.6<(V-I)_{\mathrm{KMT}}<0.2\right)$, which are a good representation of the bulge star population, to derive an instrumental $V I L$ colorcolor relation. Since the source is significantly bluer than this range we apply a new colorcolor method. For each giant we use the dereddened $(V-I)_{0}$ color to find the corresponding $(I-L)_{0}$ based on Bessell \& Brett (1988) giants, and derive the offset between the instrumental $\left(I_{\mathrm{KMT}}-L_{\text {Spitzer }}\right)$ and the $(I-L)_{0}$ for the sample. We then use the source $(V-I)_{s, 0}$ color and find its corresponding $(I-L)_{s, 0}$ based on Bessell \& Brett (1988) dwarf 1 . Finally, we use the color offset to find the source instrumental color, $\left(I_{\mathrm{KMT}}-L_{\text {Spitzer }}\right)_{s}=0.78 \pm 0.03$, which is used to constrain the Spitzer source-star flux in the modeling. For comparison, we also derive the source instrumental color using linear VIL color-color relation using the giants and extrapolate it to the source (see Calchi Novati et al. 2015b for didactic explanation). The color we find using this method is $\left(I_{\mathrm{KMT}}-L_{\text {Spitzer }}\right)_{s}=0.77 \pm 0.05$, which is in agreement with the new method. While the previous method works extremely well for colors in the range of the giants, it is important to use the new method when extrapolating from the calibration range.

\subsection{An Earth-mass planet around an ultracool dwarf}

As mentioned above, the four-fold degenerate parallax solutions $\left(\boldsymbol{\pi}_{\mathrm{E}}\right)$ have approximately the same magnitude, and so give similar masses, distance from Earth and relative proper motion of the planetary system. In addition, the wide and close solutions are very close to $s=1$, and so the projected separation is also similar. In summary, the derived physical properties of the eight degenerate model solutions are all in agreement within $1 \sigma$. Therefore, we combine the results from the different models and give the median and $68 \%$ uncertainty range of the physical properties of the system in Table 2.

The host star mass is $0.078_{-0.012}^{+0.016} M_{\odot}\left(\right.$ or $\left.81_{-13}^{+17} M_{J}\right)$, i.e., with mean just at the hydrogenburning mass limit at Solar metallicity. The companion mass is $1.43_{-0.32}^{+0.45} M_{\oplus}$, which is the lowest mass planet discovered by microlensing, and it orbits the ultracool dwarf at a projected separation of $1.16_{-0.13}^{+0.16} \mathrm{AU}$. The system lies in the Galactic disk, at $3.91_{-0.46}^{+0.42} \mathrm{kpc}$ toward the bulge.

The relative proper motion between the source and the lens is $\mu_{\text {hel }}=8.7_{-1.2}^{+1.6}$ mas $/ \mathrm{yr}$,

\footnotetext{
${ }^{1}$ Note that while the Bessell \& Brett (1988) VIL relations are derived for ground-based $L$ band, which differs somewhat from the Spitzer $3.6 \mu \mathrm{m}$ band, it can still be used essentially unmodified for our purposes. This is because these authors report $(K-L) \propto 0.04(V-K)$, which is already quite small, and the further correction from $L$ to $3.6 \mu \mathrm{m}$ is an order of magnitude smaller.
} 
which is consistent with expectations for disk lenses. However, the direction of the proper motion is clustered at $\mu_{\text {hel }}(\mathrm{N}, \mathrm{E}) \approx( \pm 4.0,-7.5) \mathrm{mas} \mathrm{yr}^{-1}$, whereas typical disk lenses at

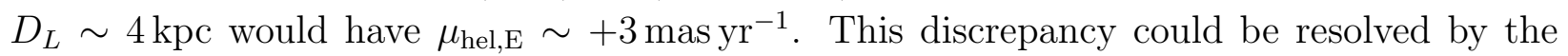
lens moving $\sim 180 \mathrm{~km} \mathrm{~s}^{-1}$ relative to its local standard of rest (i.e., halo lens) or the source moving $\sim 360 \mathrm{~km} \mathrm{~s}^{-1}$ relative to the bulge (or some combination). Both possibilities would seem to have low prior probability. However, recall that in Section 3.2 we showed that the negative value of $\pi_{\mathrm{E}, \mathrm{E}}$ (hence $\mu_{\mathrm{E}}$ ) is a direct consequence of the relatively high magnification as seen by Spitzer one week after $t_{0, \oplus}$, which is attested to by a whole series of Spitzer measurements. Hence, it is not easily avoided. The issue can be resolved by two epochs of high resolution imaging, which would measure the source proper motion relative to the bulge, $\boldsymbol{\mu}_{S}$, and so yield directly the lens proper motion $\boldsymbol{\mu}_{L}=\boldsymbol{\mu}_{S}+\boldsymbol{\mu}_{\mathrm{hel}, \mathrm{rel}}$.

\section{Discussion}

We have detected and characterized an Earth-mass planet orbiting an ultracool dwarf (at the hydrogen-burning limit, assuming Solar metallicity) at $\sim 1$ AU. This system adds to two previous microlensing discoveries of planets of a few Earth masses orbiting ultracool dwarfs. These suggest that the protoplanetary disks of ultracool dwarfs have sufficient mass to form terrestrial planets, as also hinted at by direct imaging of such disks. The location of these planets, at about $1 \mathrm{AU}$, support planet formation predictions. However, since the sensitivity of current microlensing surveys for systems with such small mass ratios is very narrow, around projected separations of $\sim 1 \mathrm{AU}$, they cannot set strong constraints on the presence of planets elsewhere around ultracool dwarfs, such as the much closer separations seen in the TRAPPIST-1 system.

The dense coverage of the short duration anomaly induced by the low mass-ratio planet OGLE-2016-BLG-1195Lb was enabled due to the high-cadence coverage of KMTNet. This shows the importance of the new high-cadence global network surveys, which do not require the traditional alert and follow up mode, previously used in microlensing. Future spacebased microlensing surveys, such as WFIRST (Spergel et al. 2015), will have this required high-cadence and with their superior photometry will extend the detection sensitivity to wider separations and lower planetary masses.

This is the third planet discovered as part of the Spitzer microlensing campaign for measuring the Galactic distribution of planets, and another few planetary candidates from the 2015 and 2016 seasons are currently being investigated. So far, all of the published planets are located in the Galactic disk. Zhu et al. (2017) studied the planet sensitivities of 2015 Spitzer events with high-cadence KMTNet coverage and found that if the frequency of planets is equal in the bulge and in the disk, about $1 / 3$ of the planet detections should be from systems in the bulge. With two final Spitzer microlensing campaigns in 2017 and 2018, 
and the expected additional planetary detections, we will be able to check for deviations from this expectation, and see if the bulge is deficient of planets, as the current detections hints and as suggested by Penny et al. (2016).

We thank D. Kirkpatrick for fruitful discussions about BDs. This work is based in part on observations made with the Spitzer Space Telescope, which is operated by the Jet Propulsion Laboratory, California Institute of Technology under a contract with NASA. This research has made use of the KMTNet system operated by the Korea Astronomy and Space Science Institute (KASI) and the data were obtained at three host sites of CTIO in Chile, SAAO in South Africa, and SSO in Australia. Work by YS and CBH was supported by an appointment to the NASA Postdoctoral Program at the Jet Propulsion Laboratory, administered by Universities Space Research Association through a contract with NASA. Work by AG, JCY and SCN were supported by JPL grant 1500811. Work by AG, WZ, YKJ and IGS were supported by NSF grant AST-1516842. Work by C.H. was supported by Creative Research Initiative Program (2009-0081561) of National Research Foundation of Korea. Work by SCN was supported by NExScI. Copyright 2017. All rights reserved.

\section{REFERENCES}

Albrow, M. D., et al. 2009, MNRAS, 397, 2099

Apai, D. 2013, Astronomische Nachrichten, 334, 57

Bennett, D. P., et al. 2008, ApJ, 684, 663

Bensby, T., et al. 2013, A\&A, 549, A147

Bessell, M. S., \& Brett, J. M. 1988, PASP, 100, 1134

Bond, I.A., Bennett, D.P., Sumi, T., et al. 2017, MNRAS, submitted, arXiv:1703.08639

Boss, A. P. 2006, ApJ, 643, 501

Calchi Novati, S., et al. 2015a, ApJ, 804, 20

-. 2015b, ApJ, 814, 92

Chauvin, G., Lagrange, A.-M., Dumas, C., Zuckerman, B., Mouillet, D., Song, I., Beuzit, J.-L., \& Lowrance, P. 2004, A\&A, 425, L29

Claret, A. 2000, A\&A, 363, 1081 
Daemgen, S., Natta, A., Scholz, A., Testi, L., Jayawardhana, R., Greaves, J., \& Eastwood, D. 2016, A\&A, 594, A83

Gauza, B., Béjar, V. J. S., Pérez-Garrido, A., Rosa Zapatero Osorio, M., Lodieu, N., Rebolo, R., Pallé, E., \& Nowak, G. 2015, ApJ, 804, 96

Gillon, M., et al. 2017, Nature, 542, 456

Gould, A. 1992, ApJ, 392, 442

-. 1994, ApJ, 421, L75

-. 2000, ApJ, 542, 785

-. 2008, ApJ, 681, 1593

Gould, A., Dong, S., Bennett, D. P., Bond, I. A., Udalski, A., \& Kozlowski, S. 2010, ApJ, 710,1800

Gould, A., \& Gaucherel, C. 1997, ApJ, 477, 580

Gould, A., \& Yee, J. C. 2012, ApJ, 755, L17

Gould, A., et al. 2014, Science, 345, 46

Griest, K., \& Safizadeh, N. 1998, ApJ, 500, 37

Han, C., et al. 2013, ApJ, 778, 38

Ida, S., \& Lin, D. N. C. 2005, ApJ, 626, 1045

Kim, S.-L., Lee, C.-U., Park, B.-G., et al. 2016, JKAS, 49, 37

Kervella, P., Thévenin, F., Di Folco, E., \& Ségransan, D. 2004, A\&A, 426, 297

Kubas, D., et al. 2012, A\&A, 540, A78

Liu, M. C., et al. 2011, ApJ, 740, 108

Lodato, G., Delgado-Donate, E., \& Clarke, C. J. 2005, MNRAS, 364, L91

Luhman, K. L. 2012, ARA\&A, 50, 65

Nataf, D. M., et al. 2013, ApJ, 769, 88

Paczynśki, B. 1986, ApJ, 304, 1 
Payne, M. J., \& Lodato, G. 2007, MNRAS, 381, 1597

Pejcha, O., \& Heyrovský, D. 2009, ApJ, 690, 1772

Penny, M. T., Henderson, C. B., \& Clanton, C. 2016, ApJ, 830, 150

Refsdal, S. 1966, MNRAS, 134, 315

Schechter, P. L., Mateo, M., \& Saha, A. 1993, PASP, 105, 1342

Spergel, D., et al. 2015, arXiv:1503.03757

Street, R. A., et al. 2016, ApJ, 819, 93

Sumi, T., et al. 2003, ApJ, 591, 204

—. 2016, ApJ, 825, 112

Testi, L., Natta, A., Scholz, A., Tazzari, M., Ricci, L., \& de Gregorio Monsalvo, I. 2016, A\&A, 593, A111

Todorov, K., Luhman, K. L., \& McLeod, K. K. 2010, ApJ, 714, L84

Udalski, A., Szymański, M. K., \& Szymański, G. 2015a, Acta Astron., 65, 1

Udalski, A., et al. 2015b, ApJ, 799, 237

Yee, J. C., et al. 2015, ApJ, 810, 155

Zhu, W., et al. 2017, ApJ, submitted, arXiv:1701.05191 
Table 1: Best-fit microlensing model parameters and their $68 \%$ uncertainty range derived from the MCMC chain density for the eight degenerate solutions

\begin{tabular}{|c|c|c|c|c|c|c|c|c|}
\hline \multirow[t]{2}{*}{ Parameter } & \multicolumn{4}{|c|}{ Close } & \multicolumn{4}{|c|}{ Wide } \\
\hline & -- & -+ & +- & ++ & -- & -+ & +- & ++ \\
\hline$t_{0}\left[\mathrm{HJD}^{\prime}\right]$ & $7568.7692 \pm 0.0013$ & $7568.7695 \pm 0.0013$ & $7568.7693 \pm 0.0013$ & $7568.7693 \pm 0.0013$ & $7568.7694 \pm 0.0013$ & $7568.7695 \pm 0.0013$ & $7568.7694 \pm 0.0013$ & $7568.7695 \pm 0.0012$ \\
\hline$u_{0}$ & $-0.05321 \pm 0.00073$ & $-0.05284 \pm 0.00079$ & $0.05317 \pm 0.00075$ & $0.05320_{ \pm} 0.00073$ & $-0.05330 \pm 0.00073$ & $-0.05321 \pm 0.00075$ & $0.05321 \pm 0.00074$ & $0.05324 \pm 0.00074$ \\
\hline$t_{\mathrm{E}}[\mathrm{d}]$ & $9.96 \pm 0.11$ & $10.01 \pm 0.11$ & $9.96 \pm 0.11$ & $9.96 \pm 0.11$ & $9.94 \pm 0.11$ & $9.95 \pm 0.11$ & $9.95 \pm 0.11$ & $9.95 \pm 0.11$ \\
\hline$\rho\left[10^{-3}\right]$ & $2.90_{-0.40}^{+0.34}$ & $2.87_{-0.41}^{+0.35}$ & $2.89_{-0.38}^{+0.34}$ & $2.90_{-0.40}^{+0.34}$ & $2.86_{-0.43}^{+0.34}$ & $2.85_{-0.42}^{+0.35}$ & $2.84_{-0.42}^{+0.35}$ & $2.85_{-0.41}^{+0.35}$ \\
\hline$\pi_{\mathrm{E}, \mathrm{N}}$ & $-0.2154 \pm 0.0065$ & $0.2335 \pm 0.0080$ & $-0.3017 \pm 0.0071$ & $0.1487 \pm 0.0074$ & $-0.2158 \pm 0.0066$ & $0.2350 \pm 0.0080$ & $-0.3016 \pm 0.0074$ & $0.1491 \pm 0.0075$ \\
\hline$\pi_{\mathrm{E}, \mathrm{E}}$ & $-0.380 \pm 0.032$ & $-0.411 \pm 0.032$ & $-0.376 \pm 0.032$ & $-0.404 \pm 0.031$ & $-0.382 \pm 0.032$ & $-0.413 \pm 0.031$ & $-0.377 \pm 0.032$ & $-0.404 \pm 0.032$ \\
\hline$\alpha[\mathrm{rad}]$ & $-0.9684 \pm 0.0022$ & $-0.9681 \pm 0.0022$ & $0.9684 \pm 0.0022$ & $0.9684 \pm 0.0022$ & $-0.9690 \pm 0.0022$ & $-0.9688 \pm 0.0022$ & $0.9688 \pm 0.0022$ & $0.9689 \pm 0.0022$ \\
\hline$s$ & $0.9842_{-0.0075}^{+0.0069}$ & $0.9834_{-0.0072}^{+0.0070}$ & $0.9839_{-0.0072}^{+0.0068}$ & $0.9840_{-0.0075}^{+0.0068}$ & $1.0856_{-0.0075}^{+0.0082}$ & $1.0861_{-0.0076}^{+0.0081}$ & $1.0862_{-0.0075}^{+0.0080}$ & $1.0853_{-0.0074}^{+0.0084}$ \\
\hline$q\left[10^{-5}\right]$ & $5.43_{-0.70}^{+0.82}$ & $5.49_{-0.73}^{+0.78}$ & $5.46_{-0.71}^{+0.81}$ & $5.47_{-0.72}^{+0.79}$ & $5.60_{-0.70}^{+0.86}$ & $5.68_{-0.73}^{+0.83}$ & $5.68_{-0.72}^{+0.80}$ & $5.58_{-0.71}^{+0.84}$ \\
\hline$\chi^{2}$ & 10214 & 10214 & 10213 & 10213 & 10214 & 10214 & 10213 & 10214 \\
\hline
\end{tabular}


Table 2: Physical properties of the planetary system

\begin{tabular}{l|c}
\hline \hline$M_{1}\left[M_{\odot}\right]$ & $0.078_{-0.012}^{+0.016}$ \\
$M_{2}\left[M_{\oplus}\right]$ & $1.43_{-0.32}^{+0.45}$ \\
$r_{\perp}[\mathrm{AU}]$ & $1.16_{-0.13}^{+0.16}$ \\
$D_{L}[\mathrm{kpc}]$ & $3.91_{-0.46}^{+0.42}$ \\
$\theta_{E}[\mathrm{mas}]$ & $0.286_{-0.038}^{+0.053}$ \\
$\mu_{\mathrm{hel}}[\mathrm{mas} / \mathrm{yr}]$ & $8.7_{-1.2}^{+1.6}$ \\
\hline \hline
\end{tabular}




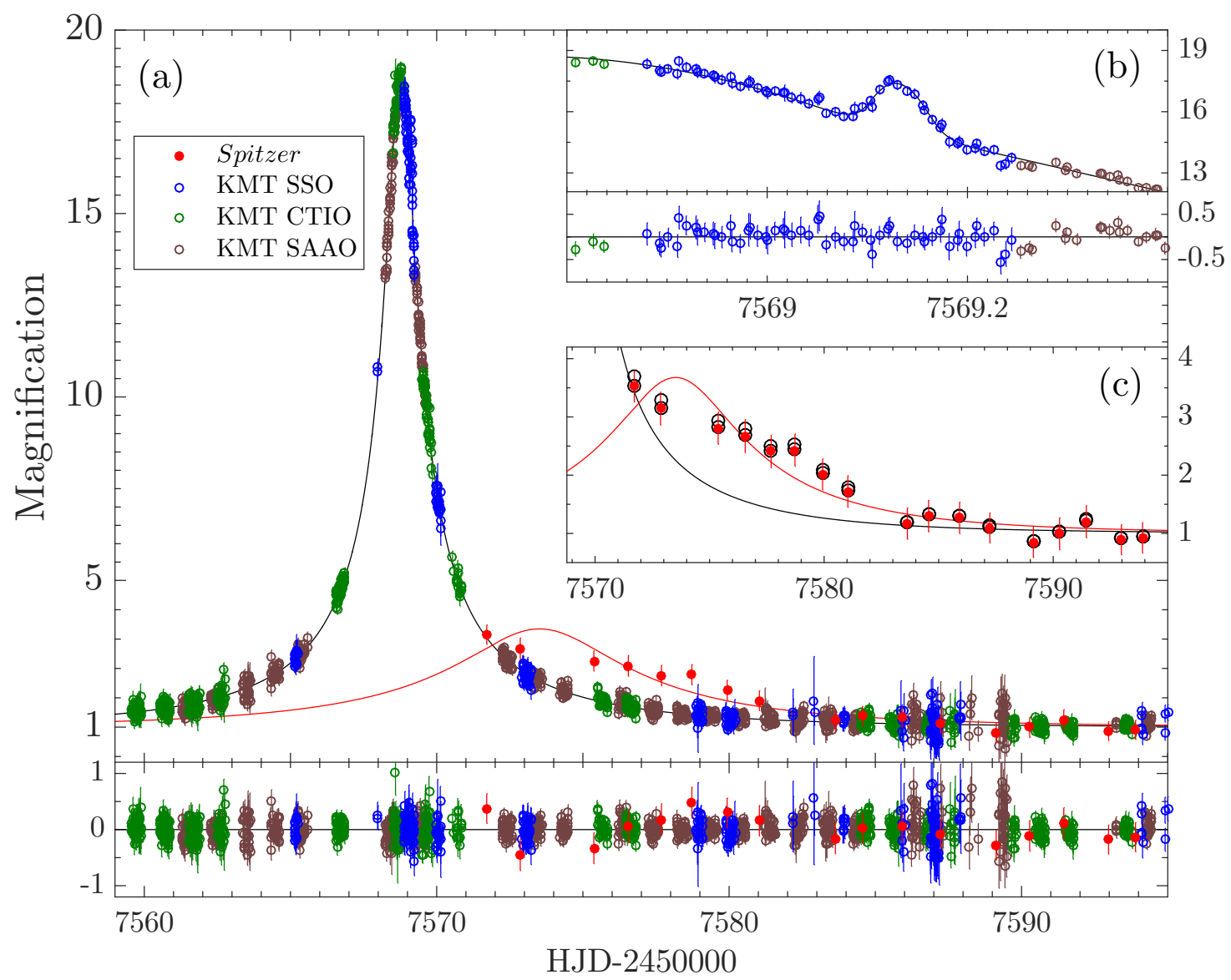

Fig. 1.- Magnification curve of OGLE-2016-BLG-1195. The short anomaly (see inset b) is well covered by KMT SSO. The microlens parallax information comes from the offset between the observed Spitzer points (red circles) and the ground curve (black line, see inset c). The open black circles are $1 \sigma$ limits on the Spitzer "observed magnification" $A$ "obs" $\equiv\left(f_{\text {obs }}-f_{\text {base }}\right) / f_{s}+1$, which are independent from the parallax model. The baseline flux $f_{\text {base }}$ can be read directly off the late-time light curve and $f_{s}$ is determined with $5 \%$ uncertainty from the VIL color-color relation and $I_{s}$, which is very accurately measured from the ground-based light curve. 

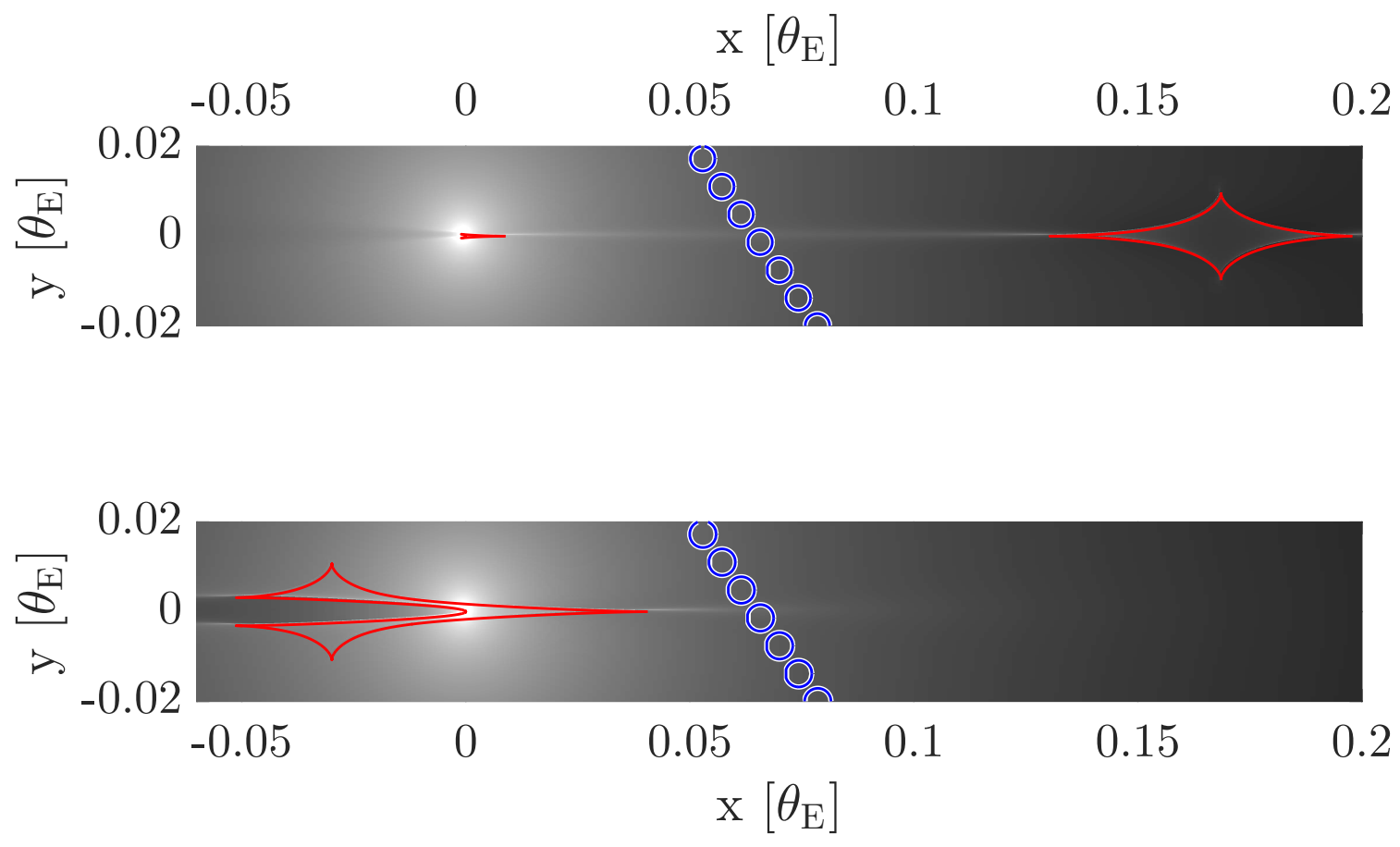

Fig. 2.- Magnification maps of the wide $(s>1$, top) and resonant $(s<1$, bottom) topologies. The source trajectory and size are represented by the blue circles. The red contours are the caustics. The gray scale indicates the magnification of a point source at each position, where white means higher magnification. 

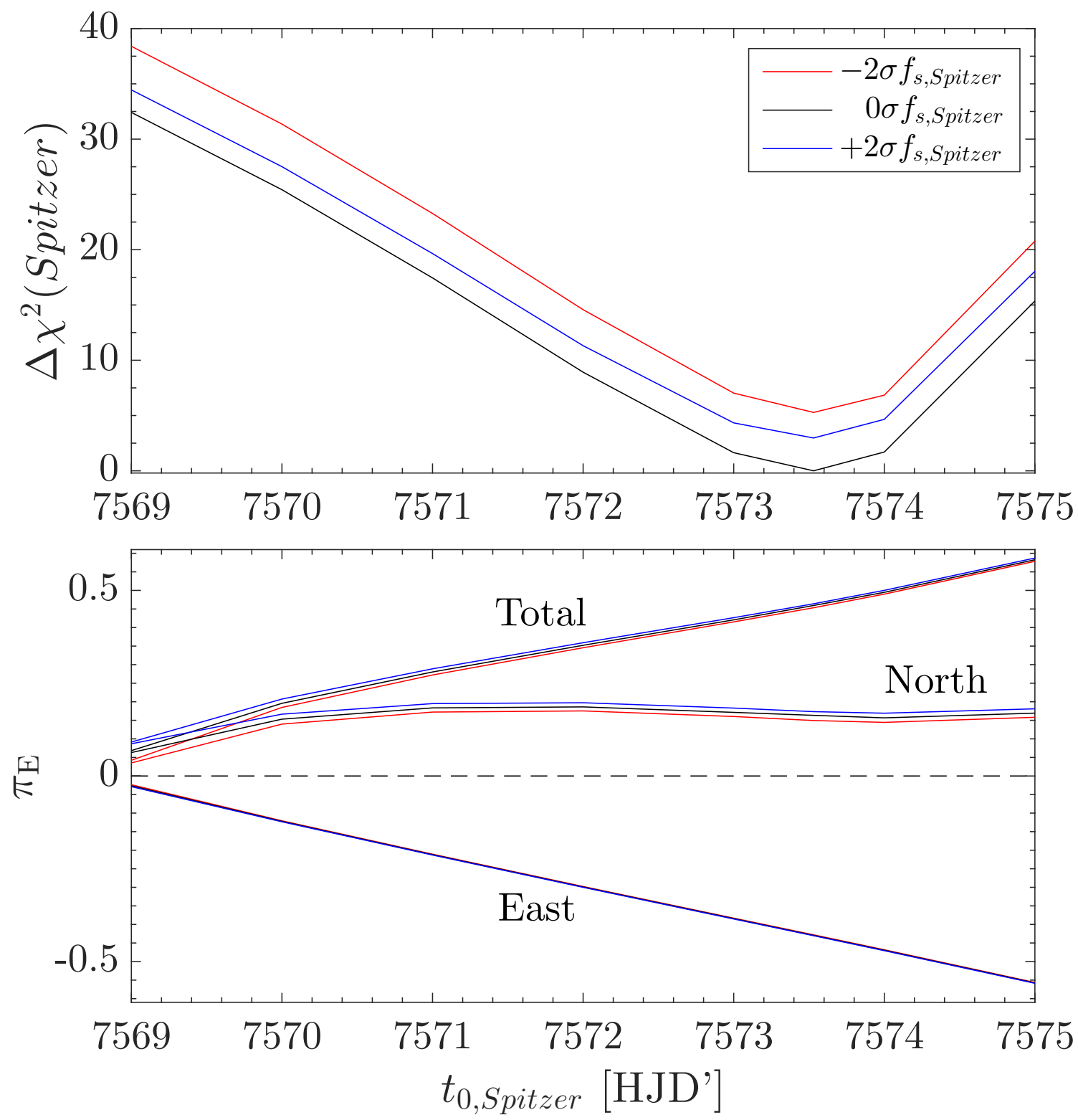

Fig. 3.- Test of robustness of microlens parallax $\boldsymbol{\pi}_{\mathrm{E}}$ measurement. Lower panel shows values of $\pi_{\mathrm{E}, \mathrm{E}}\left(\right.$ bottom), $\pi_{\mathrm{E}, \mathrm{N}}$ (middle) and total $\pi_{\mathrm{E}}$ (top) for the close $(++)$ solution when $t_{0, \text { Spitzer }}$ is forced to different values (abscissa). Red, black and blue tracks show respectively the $(-2,0,+2) \sigma$ deviations from the best estimate of $f_{s, \text { Spitzer }}$ derived from the VIL color-color constraint. These are barely distinguishable. Other $(++)$ and $(--)$ solutions are extremely similar and the $(+-)$ and $(-+)$ solutions are broadly similar but with much larger parallax at low $t_{0, \text { Spitzer }}$ (as expected from their geometry). The upper panel displays $\Delta \chi^{2}$ relative to the best fit. The figure shows that $\pi_{\mathrm{E}}$ can be forced down substantially only by forcing $t_{0, \text { Spitzer }}<7571$, where $\Delta \chi^{2}$ is already very high. See text for physical explanation of this robustness. 


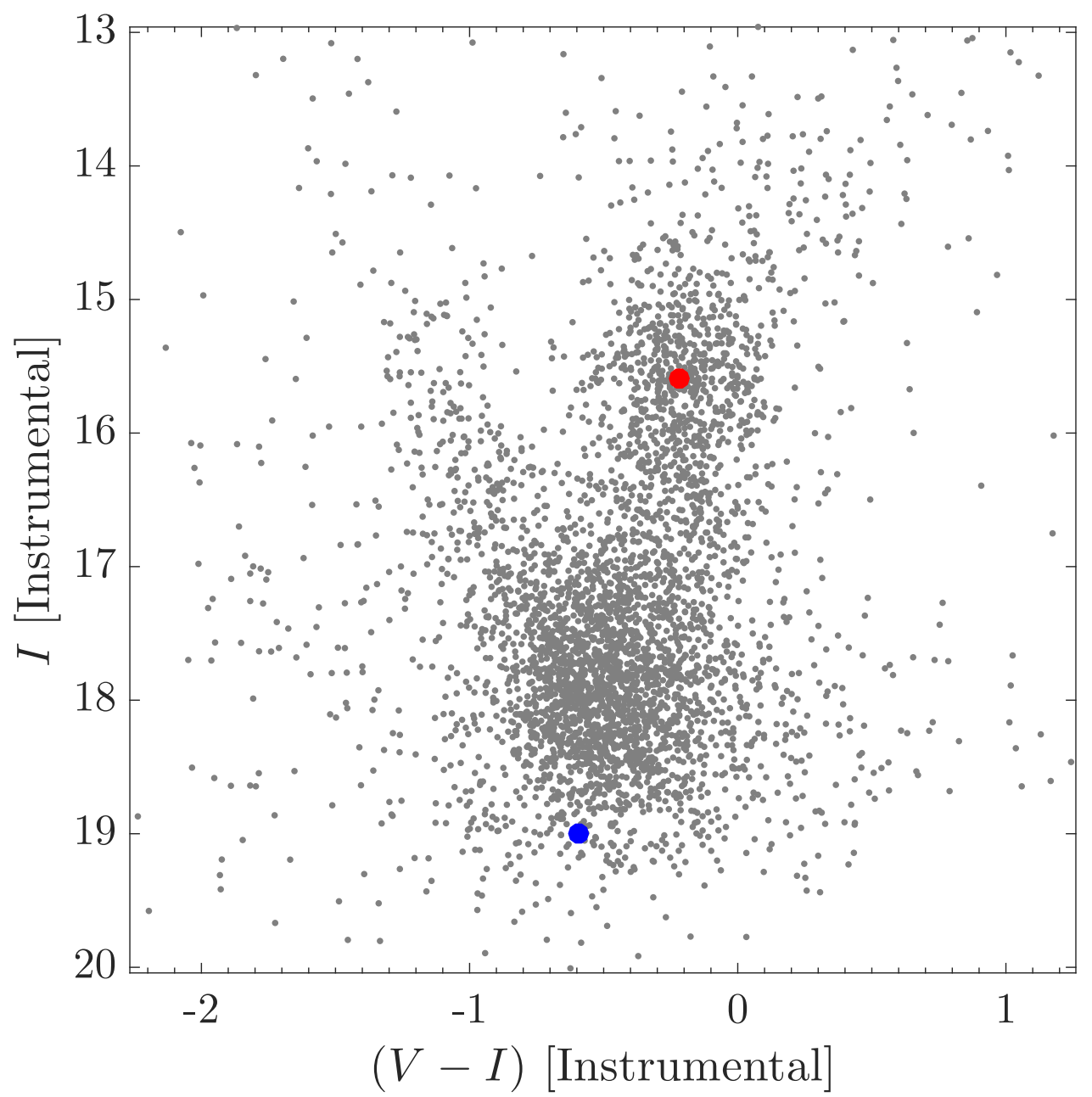

Fig. 4.- KMTNet instrumental CMD of stars from a $205^{\prime \prime} \times 205^{\prime \prime}$ field centered on the event's position. The offset between the red clump centroid (red) and the source star (blue) allows us to derive the source angular radius $\theta_{*}$. 